\title{
No difference in diagnostic yield between D- and E-speed radiographs
}

Ricketts D N J, Whaites EJ, Kidd EAM, Brown J E, Wilson R F. An evaluation of the diagnostic yield from bitewing radiographs of small approximal and occlusal carious lesions in a low prevalence sample in vitro using different film types and speeds. Br Dent J 1997; 182: 51-58

Objective To compare the diagnostic yield for the diagnosis of approximal and occlusal caries using D- and E-speed films.

Design A laboratory study in a UK dental school 1992-1994.

Materials and methods 96 extracted teeth with occlusal and approximal caries positioned in acrylic arch trays to simulate a normal anatomical relationship. Bitewing radiographs were taken using a standardised method and examined at random by 5 independent examiners for caries. All teeth were subsequently sectioned and examined histologically to validate the diagnosis.

Results E-speed films from both manufacturers required lower exposure times to produce 'subjective' gold standard images. Sensitivities and specificities for all diagnostic levels (D1-D4) and both molar and premolar teeth are given in the article. See tables 1 and 2 for examples (likelihood ratios have been calculated from data in the paper). Intra-examiner agreement is given as percentage of exactly reproduced diagnoses. Subjectively the majority of examiners ranked Agfa double packed D-speed film best.

Conclusions There was considerable diagnostic variation between the examiners but no significant differences in the diagnostic yield between the different film types tested.

Address D N J Ricketts, Guy's Hospital Dental School, London SE1 9RT, UK

\section{Commentary}

This study was unusually well reported, allowing independent analysis and review to be carried out. Meticulous attention to detail is apparent in the methodology, but some care must be taken in extrapolating directly from the laboratory to the surgery. For example, the great care taken by the researchers to optimise and equalise the optical density of the different film types and to view them under optimal conditions may not equate to the practice setting where exposure, processing and viewing standards vary. There is also potential confusion with regard to what constitutes a 'small lesion', since the study was carried out on a variety of lesion sizes including cavitation - comparison with other reference studies using the term differently will be problematic.

The low sensitivity values reported, eg a mean of $13 \%$ for dentine caries (D3 level) with all film types at both approximal and occlusal sites, is notable and is consistent with the work of others. The sensitivity of radiographs is better than that of visual caries diagnosis alone, ${ }^{1}$ but the low values do reflect the need for better diagnostic tools.

A key point was that the difference in diagnostic accuracy achieved by different dentists was greater than differences achieved with different film types. The lack of any significant differences between $\mathrm{D}$ - and E-speed films is consistent with the findings of other workers and is important given the reduction in radiation dose which can be achieved. However, it must also be appreciated that the numbers of teeth involved in this study, when analysed by tooth type, surface and depth of lesion, were so reduced that any difference between film types would have to have been large to be detected. It may have been informative to have performed the analysis with all surfaces considered together.

The authors point out in their concluding remarks that the film types used in their study have now been superseded by newer ones. It is the paradox of Evidence-based Health Care that, just as valuable evidence is provided to help the clinical team, new methods or technology evolve resulting in the need for further research. In the mean time, the research presented supports the conclusion that E-speed film is recommended for use in general practice.

1 Lussi A. Comparison of different methods for the diagnosis of fissure caries without Cavitation. Caries Res 1993; 27: 409-416.

Gail V. A. Topping ${ }^{1}$ and Nigel B. Pitts ${ }^{2}$ ${ }^{1}$ MRC Special Training Fellow, ${ }^{2}$ Professor \& Director, Dental Health Services Research Unit, Dundee, UK 